\title{
Photon polarization in the two-photon decay of heavy hydrogen-like ions
}

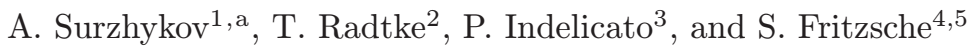 \\ 1 Physikalisches Institut, Universität Heidelberg, 69120 Heidelberg, Germany \\ 2 Institut für Physik, Universität Kassel, 34132 Kassel, Germany \\ 3 Laboratoire Kastler Brossel, École Normale Supérieure, CNRS et Université, Pierre et Marie \\ Curie-Paris 6, Case 74, 4 place Jussieu, 75005 Paris, France \\ 4 Gesellschaft für Schwerionenforschung (GSI), 64291 Darmstadt, Germany \\ ${ }^{5}$ Max-Planck-Institut für Kernphysik, 69117 Heidelberg, Germany
}

\begin{abstract}
We have applied the density matrix and second-order perturbation theory in order to re-analyze the two-photon decay of hydrogen-like ions for the polarization of emitted light. Special attention is paid to the linear polarization of one of the photons, while the spin state of the second photon is supposed to be unobserved. For such an "angle-polarization" correlation of the two photons we investigate the contributions that arise from non electric-dipole terms in the expansion of the electron-photon interaction. Detailed calculations are performed for the $2 s_{1 / 2} \rightarrow 1 s_{1 / 2}$ and $3 d_{5 / 2} \rightarrow 1 s_{1 / 2}$ transitions in neutral hydrogen as well as in hydrogen-like uranium.
\end{abstract}

\section{Introduction}

Since the seminal work of Göppert-Mayer [1], the two-photon decay of one- and few-electron atoms has been the subject of intense studies, both by experiment [2-4] and theory [5-12]. For many years, these investigations have dealt not only with the total and energy-differential transition rates but also with the angular correlations between the emitted photons. Today, much of the present interest is focused on the polarization properties of the photons whose quantum correlations may reveal unique information about the electronic structure of the ions as well as about the entanglement in such systems [13-15]. Up to the present, however, most investigations on the polarization of the correlated photons have been carried out for the (twophoton) decay of neutral hydrogen or low- $Z$ ions. Less attention, in contrast, has been paid so far to the high- $Z$ domain. First experiments on the polarization of the x-ray photons from heavy ions is likely to be carried at the GSI in Darmstadt where significant progress has been made recently in the development of x-ray polarization detectors [16,17]. By making use of these detectors it will become possible in the near future to measure not only (i) the polarization of only one photon that is observed in coincidence with the other one but also (ii) the correlated spin state of the two photons (i.e. the polarization entanglement).

In this contribution, we apply the density matrix theory to re-analyze the two-photon decay of hydrogen-like ions for the polarization properties of the emitted photons. Although the density matrix approach provides an efficient way to treat both, the (i) "angle-polarization" and (ii) "polarization-polarization" (i.e. entanglement) two-photon correlations mentioned above, here we restrict ourselves to the first scenario in which the polarization properties of one photon is analyzed, provided that the second photon is recorded under a given direction but without

\footnotetext{
a e-mail: surz@physi.uni-heidelberg.de
} 
that its spin state is observed. For such a simplified two-photon polarization scenario, the basic relations are discussed in Section 2. There, we also give a brief account on the (relativistic) second-order perturbation theory that is utilized to calculate the two-photon transition amplitudes. Making use of these amplitude, we then derive the spin density matrix for one of the emitted photons and parameterize such a matrix in terms of polarization Stokes parameters. For the $2 s_{1 / 2} \rightarrow 1 s_{1 / 2}$ and $3 d_{5 / 2} \rightarrow 1 s_{1 / 2}$ two-photon transitions, moreover, we also provide simplified expressions for the Stokes parameters as obtained within the non-relativistic dipole approximation. In Section 3, we employ these expressions together with the results from our rigorous relativistic calculations in order to analyze the polarization properties of the two-photon decay of neutral hydrogen and hydrogen-like uranium. These computations demonstrate that, for high- $Z$ ions, the higher multipoles in the expansion of the electron-photon interaction lead to a slight reduction of the linear polarization of the emitted photons; an effect which becomes important only for unequal energy sharing between the two photons. Finally, a brief summary is given in Section 4.

\section{Theoretical background}

\subsection{Two-photon transition amplitude}

Not much has to be said about the basic formalism for studying the two-photon decay in hydrogen-like ions. In the past, this formalism has been widely applied to explore not only the total decay rates $[6,10]$ but also the angular correlations of the emitted photons [11]. Usually, the analysis of these properties can be traced back to the evaluation of the bound-bound transitions amplitude which, in second-order perturbation theory, is given by:

$$
\begin{aligned}
M_{f i}\left(\mu_{f}, \mu_{i}, \lambda_{1}, \lambda_{2}\right)= & \sum_{\nu} \frac{\left\langle\psi_{n_{f} j_{f} \mu_{f}}\left|\alpha \mathbf{u}_{\lambda_{1}}^{*} e^{-i \boldsymbol{k}_{1} \boldsymbol{r}}\right| \psi_{\nu}\right\rangle\left\langle\psi_{\nu}\left|\alpha \mathbf{u}_{\lambda_{2}}^{*} e^{-i \boldsymbol{k}_{2} \boldsymbol{r}}\right| \psi_{n_{i} j_{i} \mu_{i}}\right\rangle}{E_{\nu}-E_{i}+E_{\gamma_{2}}} \\
& +\sum_{\nu} \frac{\left\langle\psi_{n_{f} j_{f} \mu_{f}}\left|\alpha \mathbf{u}_{\lambda_{2}}^{*} e^{-i \boldsymbol{k}_{2} \mathbf{r}}\right| \psi_{\nu}\right\rangle\left\langle\psi_{\nu}\left|\alpha \mathbf{u}_{\lambda_{1}}^{*} e^{-i \boldsymbol{k}_{1} \mathbf{r}}\right| \psi_{n_{i} j_{i} \mu_{i}}\right\rangle}{E_{\nu}-E_{i}+E_{\gamma_{1}}} .
\end{aligned}
$$

In this expression, the transition operator $\alpha \mathbf{u}_{\lambda_{i}} e^{i \boldsymbol{k}_{i} \boldsymbol{r}}$ describes the (relativistic) electron-photon interaction and the unit vectors $\mathbf{u}_{\lambda_{i}}$ the polarization of the individual photons. Within the relativistic theory, moreover, the energies $E_{i}$ and $E_{f}$ and the wave functions $\psi_{n_{i} j_{i} \mu_{i}}(\boldsymbol{r})$ and $\psi_{n_{f} j_{f} \mu_{f}}(\boldsymbol{r})$ are obtained from Dirac's equations for a singly bound electron in the field of the nucleus [18]. Because of energy conservation, $E_{i}$ and $E_{f}$ are related to the energies $E_{\gamma_{1,2}}$ of the emitted photons by:

$$
E_{i}-E_{f}=E_{\gamma_{1}}+E_{\gamma_{2}}
$$

From this relation, it is convenient to define the so-called energy sharing $x=E_{\gamma_{1}} /\left(E_{\gamma_{1}}+E_{\gamma_{2}}\right)$, i.e. the fraction of the energy which is carried away by the "first" photon.

The summation over the intermediate states in the second-order transition amplitude (1) runs over the complete one-particle spectrum $\psi_{\nu}(\boldsymbol{r})$, including a summation over the discrete part of the spectrum as well as an integration over the positive and negative-energy continuum. Apart from the direct summation as implemented, for example, by the B-spline finite basis set approach [10], the amplitudes (1) can be evaluated also by means of the Green's function of the ion. This latter method is particularly helpful for hydrogen-like ions, since the CoulombGreen's functions are known analytically, both within the nonrelativistic as well as relativistic theory $[19,20]$.

\subsection{Density matrix of the emitted photons}

Equation (1) displays the general form of the relativistic transition amplitude for the twophoton decay of hydrogen-like ions. In the following, we shall employ this amplitude together 
with the Green's function approach in order to analyze the polarization properties of the emitted radiation. Most naturally, such an analysis can be performed by means of the density matrix that is associated to the spin-polarization of the emitted photons. Since the application of the density matrix formalism to the two-photon decay has been discussed at several places elsewhere [15,21], here we just display the helicity representation of the (two-photon) spin density matrix in its final form:

$$
\left\langle\boldsymbol{k}_{1} \lambda_{1}, \boldsymbol{k}_{2} \lambda_{2}\left|\hat{\rho}_{f}\right| \boldsymbol{k}_{1} \lambda_{1}^{\prime}, \boldsymbol{k}_{2} \lambda_{2}^{\prime}\right\rangle=\frac{1}{2 j_{i}+1} \sum_{\mu_{i}, \mu_{f}} M_{f i}\left(\mu_{f}, \mu_{i}, \lambda_{1}, \lambda_{2}\right) M_{f i}^{*}\left(\mu_{f}, \mu_{i}, \lambda_{1}^{\prime}, \lambda_{2}^{\prime}\right),
$$

and where $\lambda_{1,2}= \pm 1$ denotes the spin projection of the photons onto their propagation direction $\boldsymbol{k}_{1,2}$.

The final-state density matrix (3) contains the complete information about the total spin state of the two emitted photons in the decay of an (unpolarized) ion in the level $\left|n_{i} j_{i}\right\rangle$. Since, in our present work, we wish to investigate the polarization of only one of the photons, the partial trace has to be taken in Eq. (3) over the unobserved spin states of the second photon:

$$
\left\langle\boldsymbol{k}_{1} \lambda_{1}\left|\hat{\rho}_{\gamma}\left(\boldsymbol{k}_{2}\right)\right| \boldsymbol{k}_{1} \lambda_{1}^{\prime}\right\rangle=\frac{1}{2 j_{i}+1} \sum_{\mu_{i}, \mu_{f}, \lambda_{2}} M_{f i}\left(\mu_{f}, \mu_{i}, \lambda_{1}, \lambda_{2}\right) M_{f i}^{*}\left(\mu_{f}, \mu_{i}, \lambda_{1}^{\prime}, \lambda_{2}\right) .
$$

This gives rise to the reduced density matrix that, however, still depends on the wave vectors of both photons $\boldsymbol{k}_{1}$ and $\boldsymbol{k}_{2}$, indicating that the polarization of the "first" photon is measured in coincidence with the "second" one.

\subsection{Polarization parameters of emitted radiation}

Within the helicity representation the reduced density matrix (4) is a $2 \times 2$ matrix and can thus be parameterized by the three real Stokes parameters [22]:

$$
\left\langle\mathbf{k}_{1} \lambda_{1}\left|\hat{\rho}_{\gamma}\left(\boldsymbol{k}_{2}\right)\right| \boldsymbol{k}_{1} \lambda_{1}^{\prime}\right\rangle=\frac{1}{2}\left(\begin{array}{cc}
1+P_{3} & P_{1}-i P_{2} \\
P_{1}+i P_{2} & 1-P_{3}
\end{array}\right) .
$$

In experiments, these parameters are often utilized for characterizing the degree of polarization of the emitted light. While the Stokes parameter $P_{3}$ reflects the circular polarization, the two parameters $P_{1}$ and $P_{2}$ together denote the degree and direction of the linear polarization of the photons. These two parameters can be determined quite easily by measuring the intensities of the light $I_{\chi}$, linearly polarized under various angles with respect to the reaction plane (as formed by the directions $\boldsymbol{k}_{1}$ and $\boldsymbol{k}_{2}$ of the two photons). While the first parameter is given by the intensity ratio $P_{1}=\left(I_{0}-I_{90}\right) /\left(I_{0}+I_{90}\right)$, the parameter $P_{2}$ follows from a similar intensity ratio, taken at $\chi=45^{\circ}$ and $\chi=135^{\circ}$, respectively.

As seen from Eqs. (4) and (5), any further analysis of the polarization Stokes parameters can be traced back to the evaluation of the second-order transition amplitude $M_{f i}\left(\mu_{f}, \mu_{i}, \lambda_{1}, \lambda_{2}\right)$. In fact, this amplitude accounts for the full interaction between the electron and the radiation field since the operator $\boldsymbol{\alpha} \boldsymbol{u}_{\lambda_{i}} e^{i \boldsymbol{k}_{i} \boldsymbol{r}}$ includes also all the non-dipole terms in the expansion of the electron-photon interaction. However, since these higher-multipole terms are often small, at least for low- and mid- $Z$ elements, it seems to be justified in many cases to treat the electron-photon interaction in the long-wave (or non-relativistic dipole) approximation by setting $e^{i \boldsymbol{k}_{i} \boldsymbol{r}} \approx 1$. This "dipole approximation" is valid if the photon wave length is much larger than the size of the atom, i.e. for $k a_{0} \ll 1$ and where $a_{0}$ is the Bohr radius. For the two-photon decay, this condition is well satisfied for most light ions with, say, $Z<30$.

Making use of the long-wave approximation we are able especially to derive an analytical expression for the reduced density matrix (4) and, hence, for the Stokes parameters of the 

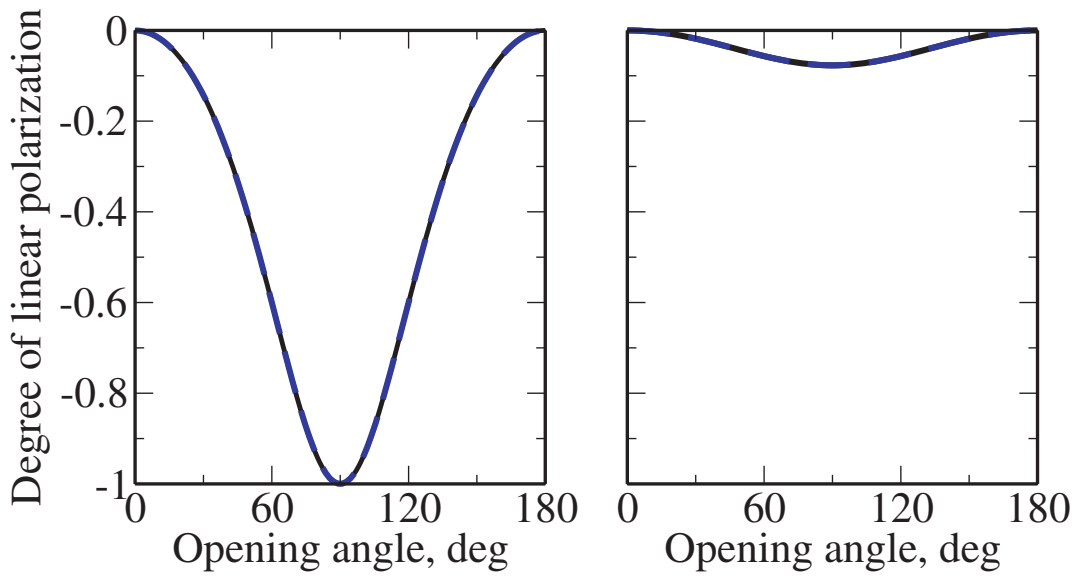

Fig. 1. Degree of linear polarization of the radiation emitted in the $2 s_{1 / 2} \rightarrow 1 s_{1 / 2}$ (left panel) and $3 d_{5 / 2} \rightarrow 1 s_{1 / 2}$ (right panel) two-photon decay of neutral hydrogen as function of the opening angle $\theta$. Results are presented for the exact relativistic theory (solid line) as well as in the non-relativistic dipole approximation (dashed line) and for an energy sharing $x=0.1$.

emitted radiation. Of course, these parameters depend not only on the opening angle $\theta$ between the emitted photons but also on the initial $\left|n_{i} j_{i}\right\rangle$ and final $\left|n_{f} j_{f}\right\rangle$ ionic states. For instance, the first Stokes parameter $P_{1}$ of the photons emitted in the $2 s_{1 / 2} \rightarrow 1 s_{1 / 2}$ E1E1 decay is given by:

$$
P_{1}(\theta)=-\frac{\sin ^{2} \theta}{1+\cos ^{2} \theta}
$$

while the same parameter for the $3 d_{5 / 2} \rightarrow 1 s_{1 / 2}$ E1E1 transition reads as:

$$
P_{1}(\theta)=-\frac{\sin ^{2} \theta}{13+\cos ^{2} \theta}
$$

In contrast, the second Stokes parameter $P_{2}$ vanishes identically for both transitions; together with the negative sign of the $P_{1}$ in Eqs. (6) and (7), this implies that the E1 photons are linearly polarized perpendicular to the reaction plane.

\section{Results and discussion}

As discussed in Section 2.3, the long-wave approximation is valid for analyzing the polarization properties of the light emitted in the two-photon decay of low- $Z$ ions. It might be questioned for medium- and high- $Z$ ions for which retardation effects on the electron-photon interaction become sizable. For the two-photon angle-angle correlation, for example, a significant change was found to arise from the higher multipoles and lead to clear deviations in the angular distribution of the two photons when compared with the (non-relativistic) dipole approximation [11].

In this work, we have employed both the electric-dipole and the exact relativistic treatment from Eq. (1) in order to investigate the relativistic and retardation effects on the linear polarization of the photons. Detailed calculations along the hydrogen isoelectronic sequence have been carried out, in particular, for the $2 s_{1 / 2} \rightarrow 1 s_{1 / 2}$ and $3 d_{5 / 2} \rightarrow 1 s_{1 / 2}$ two-photon transitions. For these two transitions, Fig. 1 displays for neutral hydrogen the Stokes parameter $P_{1}$ of the low-energy photon, measured in coincidence with a high-energy photon under the opening angle $\vartheta$. Computations were performed within the dipole approximation (dashed line) as well as the exact relativistic treatment (solid line) which includes all allowed multipole components in the electron-photon interaction. As expected for ions near the neutral end of the sequence, both approximations basically coincide and, hence, are well described by Eqs. (6) and (7). That 

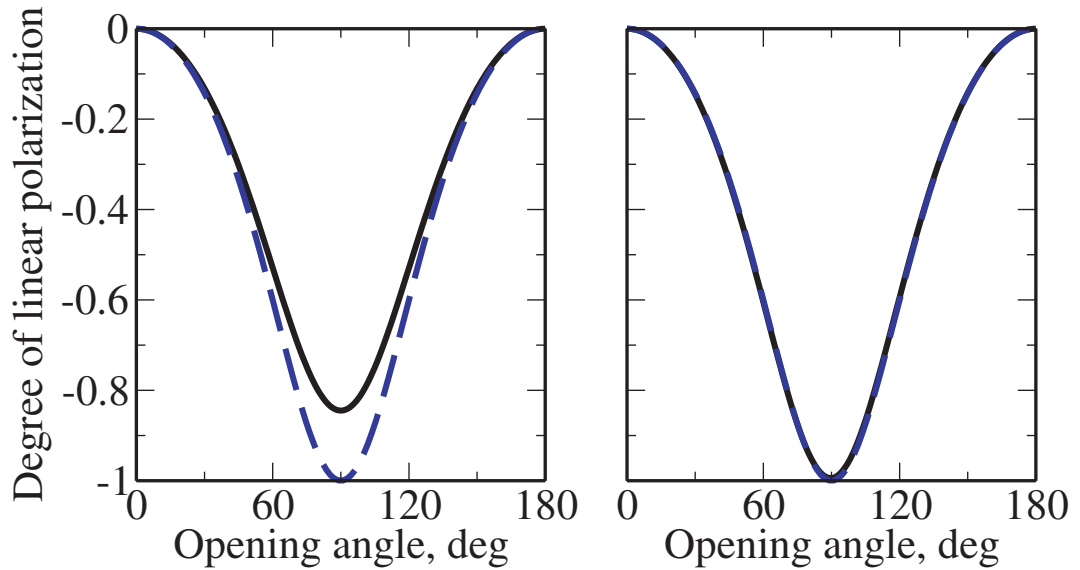

Fig. 2. Degree of linear polarization of the radiation emitted in the $2 s_{1 / 2} \rightarrow 1 s_{1 / 2}$ two-photon decay of hydrogen-like uranium $\mathrm{U}^{91+}$ as a function of the opening angle $\theta$. Results are presented for the exact relativistic theory (solid line) as well as in the non-relativistic dipole approximation (dashed line) and for an energy sharing $x=0.1$ (left panel) and $x=0.3$ (right panel).

is, the (absolute value of the) linear polarization is maximal for the opening angle $\theta=90^{\circ}$ and vanishes identically for the parallel $\left(\theta=0^{\circ}\right)$ and back-to-back $\left(\theta=180^{\circ}\right)$ photon emission. Such a depolarization results from the fact that the two-photon emission in either parallel or anti-parallel direction does not break the axial symmetry for the collision system and, hence, does not enable one to define uniquely the reaction plane.

While the long-wave and exact relativistic treatment almost coincide for the two-photon decay of neutral hydrogen, they start to differ as the nuclear charge $Z$ is increased. As seen from Fig. 2, the non-dipole terms may result in a reduction of the linear polarization of the low-energy photon emitted in the $2 s_{1 / 2} \rightarrow 1 s_{1 / 2}$ two-photon decay of hydrogen-like $\mathrm{U}^{91+}$ ions. However, such a reduction can be observed only if one of the photons is much more energetic than the second one, i.e. for either $E_{\gamma_{1}} \gg E_{\gamma_{2}}$ or $E_{\gamma_{2}} \gg E_{\gamma_{1}}$. For the energy sharing $x=0.1$ and for the perpendicular photon emission $\left(\theta=90^{\circ}\right)$, for example, the degree of linear polarization decreases from $P_{L}=-1$ to almost $P_{L}=-0.8$ if the higher multipoles are taken into account. The non-dipole effects almost disappear for a nearly equal energy sharing $E_{\gamma_{1}} \approx E_{\gamma_{2}}$ of the two photons (or $x \approx 0.5$ ). For a energy sharing of $x=0.3$, especially, the two approximations do not differ by more than $0.5 \%$. Since the photons are more likely emitted with nearly the same energy [6], our numerical results imply that the simple analytical expression (6) from the (non-relativistic) long-wave limit can be applied also for the analysis of the forthcoming experiments that are planned on the polarization properties of two-photon x-ray emission from high- $Z$ ions.

\section{Summary}

In summary, the two-photon decay of hydrogen-like ions has been re-investigated in the framework of the density matrix theory with emphasis on the polarization properties of emitted radiation. Special attention was paid to the (degree of) linear polarization of one of the photons measured in coincidence with the second photon whose polarization remains unobserved. Simple analytical expressions have been derived for such an "angle-polarization" correlation if observed in the $2 s_{1 / 2} \rightarrow 1 s_{1 / 2}$ and $3 d_{5 / 2} \rightarrow 1 s_{1 / 2}$ two-photon transitions. Although these expressions were obtained within the non-relativistic dipole approximation, they can also be applied for polarization studies with high- $Z$ ions. For these ions, the higher non-dipole terms in the electron-photon interaction were found to be of minor importance for the linear polarization of the emitted photons. 
The work of A.S. was supported by Helmholtz Gemeinschaft (Nachwuchsgruppe VH-NG-421). S.F. acknowledges support by GSI in the project KS-FRI.

\section{References}

1. M. Göppert-Mayer, Ann. Phys. (Leipzig) 9, 273 (1931)

2. R. Marrus, R.W. Schmieder, Phys. Rev. A 5, 1160 (1972)

3. D. O'Connell, K.J. Kollath, A.J. Duncan, H. Kleinpoppen, J. Phys. B 8, L214 (1975)

4. P.H. Mokler, R.W. Dunford, Phys. Scr. 69, C1 (2004)

5. G. Breit, E. Teller, Astrophys. J. 91, 215 (1940)

6. S.P. Goldman, G.W.F. Drake, Phys. Rev. A 24, 183 (1981)

7. G.W.F. Drake, S.P. Goldman, Phys. Rev. A 23, 2093 (1981)

8. V. Florescu, Phys. Rev. A 30, 2441 (1984)

9. V. Florescu, S. Patrascu, O. Stoican, Phys. Rev. A 36, 2155 (1987)

10. J.P. Santos, F. Parente, P. Indelicato, Eur. Phys. J. D 3, 43 (1998)

11. A. Surzhykov, P. Koval, S. Fritzsche, Phys. Rev. A 71, 022509 (2005)

12. U.D. Jentschura, A. Surzhykov, Phys. Rev. A 77, 042507 (2008)

13. A. Aspect, J. Dalibard, G. Roger, Phys. Rev. Lett. 49, 1804 (1982)

14. H. Kleinpoppen, A.J. Duncan, H.-J. Beyer, Z.A. Sheikh, Phys. Scr. T 72, 7 (1997)

15. T. Radtke, A. Surzhykov, S. Fritzsche, Phys. Rev. A 77, 022507 (2008)

16. S. Tashenov, Th. Stöhlker, D. Banaś, K. Beckert, P. Beller, H.F. Beyer, F. Bosch, S. Fritzsche, A. Gumberidze, S. Hagmann, C. Kozhuharov, T. Krings, D. Liesen, F. Nolden, D. Protic, D. Sierpowski, U. Spillmann, M. Steck, A. Surzhykov, Phys. Rev. Lett. 97, 223202 (2006)

17. S. Fritzsche, P. Indelicato, T. Stöhlker, J. Phys. B 38, S707 (2005)

18. J. Eichler, W.E. Meyerhof, Relativistic Atomic Collisions (Academic, San Diego, 1995)

19. R.A. Swainson, G.W.F. Drake, J. Phys. A: Math. Gen. 24, 95 (1991)

20. A. Surzhykov, P. Koval, S. Fritzsche, Comput. Phys. Commun. 165, 139 (2005)

21. L. Borowska, A. Surzhykov, Th. Stöhlker, S. Fritzsche, Phys. Rev. A 74, 062516 (2006)

22. V.V. Balashov, A.N. Grum-Grzhimailo, N.M. Kabachnik, Polarization and Correlation Phenomena in Atomic Collisions (Kluwer Academic Plenum, New York, 2000) 